\section{Effect of Fitness Training Encompassing Gymnastics-Oriented Program of Six Weeks Duration on Performance of Volleyball Players}

\author{
Syed Ibrahim ${ }^{1 *}$, Moataz Hassanain ${ }^{1}$, Syed Azhar Ahmed ${ }^{2}$, Syed Muneer Ahmed ${ }^{3}$ and Syed Kaleem Ahmed \\ ${ }^{1}$ King Fahd University of Petroleum and Minerals, Dhahran, Saudi Arabia \\ ${ }^{2}$ Ratna Physiotherapy College, Hyderabad, Telangana, India \\ ${ }^{3}$ Greater Hyderabad Municipal Corporation, Hyderabad, Telangana, India \\ ${ }^{4}$ Independent Researcher, Hyderabad, Telangana, India
}

*Corresponding author: Syed Ibrahim, King Fahd University of Petroleum and Minerals, Dhahran 34463, Saudi Arabia, Tel: +966 506137368; Fax: +966 506137368; Email: sibrahim@kfupm.edu.sa

Received date: December 12, 2017; Accepted date: January 16, 2018; Published date: February 05, 2018

Copyright: (C) 2018 Ibrahim S, et al. This is an open-access article distributed under the terms of the Creative Commons Attribution License, which permits unrestricted use, distribution and reproduction in any medium, provided the original author and source are credited.

\begin{abstract}
The main purpose of the present study was to find out the effect of six weeks of gymnastic oriented training program on the physical fitness and the level of technical performance of Volleyball players. A group of 27 Volleyball players were divided into 12 experimental and 15 control groups and their age ranged between 18 to 24 years. Physical Fitness tests comprising of vertical jump, push-ups, chin ups, sit ups, back strength, $30 \mathrm{~m}$ sprint, boomerangs test, $600 \mathrm{~m}$ run, sit and reach, bridge up and shoulder rotation and technical skill tests like passing, spiking and service were used for the study. A pre and post test was conducted before starting the training program and after the 6 weeks. It was found that both the physical fitness and skill level of the Volleyball players increased through the participation in the gymnastics oriented program.
\end{abstract}

Keywords: Gymnastic; Physical fitness; Technical skill tests; Elite player; Volleyball

\section{Introduction}

It is a global phenomenon that fitness is a pre-requisite to the desired end of optimum efficiency in elite class Volleyball players which can be achieved through appropriate conditioning program [1]. It is an accepted fact that the discipline of Gymnastics is a mother sport. The term Gymnastics dates back to the Greek culture, who used it for all kinds of physical activities like running, jumping, climbing, throwing, wrestling, boxing, chariot racing etc. Later the concept of Gymnastics underwent innumerable changes and evolved the present day Gymnastics including Artistic and Rhythmic Gymnastics [2]. There are a wide range of activities with or without apparatus which fall under the family of Gymnastics. The sport is based on man's primary movements like running, jumping, climbing, swinging, tumbling, balancing, revolving, twisting, pirouetting, throwing etc. It is a foregone conclusion that almost all the sports discipline and events use them over demanding situations all over the world [3]. An athlete's fitness is enhanced with selected gymnastic exercises with or without apparatus. Keeping this fact in mind almost all the athletes irrespective of the game participate and learn gymnastic exercises. Sports scientists and coaches agree that fitness is considered as one of the prime factors responsible for high performance in any sports activity. The domain of sports and games encompasses and places a special status to fitness. Coe et al. clearly defines that the heath related components of physical fitness comprise of cardio-respiratory fitness, muscular strength and endurance, body composition and flexibility [4]. The motor components of fitness classified are power, speed, agility, balance, reaction time and coordination. Hence it is imperative that a good training program should emphasize on activities aimed at improving all the components of physical fitness and gymnastics exercise aid in the development of these qualities. According to Trevor Dowdell perhaps gymnastics has more to offer than any other single activity towards an all-round development of physical fitness. Heinen et al. [5], laid emphasis on learning as a combination of tumbling skills to enhance psycho-physiological factors as strength, agility, endurance, flexibility, coordination, kinesthetic perception, courage and selfconfidence. George and Paschalis stated, that the student's physical or motor attributes can be enhanced through the use of gymnastics and that athletes can supplement their training with gymnastic exercises [6].

Volleyball is a popular fast paced game with many moving skills. A volleyball player has to perform many moves in multiple directions by running, scuffing, side stepping, cross stepping, pivoting, jumping and combination of other movements. It has been pointed that the game requires strength, speed, endurance, flexibility, agility and coordination along with the skills for performing well in competitions [7]. Therefor it is imperative that fitness forms a pre-requisite to the desired end of optimum efficiency in elite volleyball teams which can be achieved with appropriate fitness conditioning regime. It has been analyzed that in the game of basketball a players indulges in 300 to 400 acts of motor movements of which 50 to $60 \%$ of jumps, 30 to $35 \%$ of dashes and 15 to $20 \%$ of defensive movements. To perform these activities a player has to possess a high amount of physical fitness, mental alertness, and exceptional precision, differentiation of movements, fast switching from one form of movements to another with different rhythm, speed and coordination. For the development of the all the above qualities there is a need to schedule the training of agility and vestibular steadiness. Cormie et al. [8] suggested complex coordinative type of exercises encompassing acrobatics and gymnastic exercise like jumps on trampoline, depth jumps, different rotational movements, possible accelerations, sharp changes in running directions with sudden stops etc. Keeping the above factors in mind 
Citation: Ibrahim S, Hassanain M, Ahmed SA, Ahmed SM, Ahmed SK (2018) Effect of Fitness Training Encompassing Gymnastics-Oriented Program of Six Weeks Duration on Performance of Volleyball Players. J Sports Med Doping Stud 8: 199. doi: 10.4172/2161-0673.1000199

Page 2 of 4

the present study was undertaken to find the effect the gymnastic oriented program on the fitness and performance level of basketball players.

\section{Materials and Methods}

Twenty seven male Volleyball players undergoing training for the Interuniversity Competition were divided into an experimental group $(n=12)$ and a control group $(n=15)$. Their age ranged between 18 to 24 years. The anthropometric measurement consisting of age, height and weight were obtained. The protocol of the Physical Fitness tests for the pre and post data collection were: Vertical jump (in centimeters for measuring explosive strength), Push-ups on floor (max. number to measure arms and shoulder strength), Chin-ups on horizontal bar (Max. number to measure arms and shoulder strength), Bent Knee Situps (Max. number in 30 seconds to measure muscular endurance), Back Dynamometer (Kilograms to measure the back strength), 30 meters dash (seconds to measure the speed), Modified Boomerang Test (Seconds to measure agility and coordination), 6000 meters run (Minutes to measure endurance), Sit and Reach test ( $\mathrm{cm}$ to measure the flexibility), Bridge up (Distancellength of the bridge in $\mathrm{cm}$ to measure trunk flexibility) and Shoulder rotation (shoulder rotation index, calculated by dividing grip width in $\mathrm{cm}$ with shoulder width in $\mathrm{cm})$. The technical tests which were evaluated were passing, spiking and service. Each of the subject performed the skill tests five times and the maximum marks allotted for the test was 15 (3 marks for each trail). The training protocol consisted of 90 minutes per session, five days a week for a period of six weeks. The experimental was subjected to specific study training while the control group participated in normal training program. The schedule of training was composed of : 1) Warming up - 10 minutes 2) Stretching and flexibility exercises- 10 minutes 3) Trampoline and Tumbling exercises - 30 minutes 4) Gymnastic Apparatus work - 20 minutes 5) Specific conditioning exercises- 15 minutes 6) Cool down- 5 minutes. The statistical tool to analyze the data for the study was paired T-Test to find the significant difference in various components between the pre and post-test training scores of the experimental group and between the pre training and scores after 6 weeks of training in case of the control group.

\section{Results}

The results related to the variables of the study are presented in the following tables below:

\begin{tabular}{|c|c|c|c|c|c|c|}
\hline \multirow{3}{*}{ S No } & \multirow{3}{*}{ Variables } & Mean \pm SD & Mean \pm SD & \multirow{3}{*}{ Mean Difference } & \multirow{3}{*}{ SEMD } & \multirow{3}{*}{ T-Value } \\
\hline & & Experimental & Control & & & \\
\hline & & $(\mathrm{N}=12)$ & $(\mathrm{N}=15)$ & & & \\
\hline 1 & Age(yr) & $28.1 \pm 4.43$ & $27.8 \pm 2.98$ & 0.4 & 1.43 & $0.28 \mathrm{NS}$ \\
\hline 2 & Height $(\mathrm{cm})$ & $176.78 \pm 5.05$ & $173.62 \pm 4.99$ & 3.16 & 1.94 & $1.63 \mathrm{NS}$ \\
\hline 3 & Weight(kg) & $66.73 \pm 6.05$ & $62.74 \pm 6.72$ & 3.99 & 2.49 & $1.60 \mathrm{NS}$ \\
\hline
\end{tabular}

Table 1: Variables involved in the study $\left({ }^{\star} \mathrm{NS}=\right.$ not significant $)$.

Table 1 clearly indicates that there exists no significant difference in the anthropometric measurements of age, height and weight between the experimental and control group as the T-Values observed were
$0.28,1.63$ and 1.60 respectively. This gives a clear credence that the selected groups are similar in their physical characteristics.

\begin{tabular}{|c|c|c|c|c|c|c|c|}
\hline s No. & Variable & Group & Mean Pre-test & $\begin{array}{l}\text { Mean post } \\
\text { test }\end{array}$ & Mean difference & SED & T-Value \\
\hline 1 & \multirow{2}{*}{ Vertical jump(cm) } & Control & 56 & 57.13 & 1.13 & 0.65 & 1.73 \\
\hline & & Experimental & 54.63 & 55.88 & 1.25 & 0.48 & 2.60 \\
\hline \multirow[t]{2}{*}{2} & \multirow{2}{*}{ Push-ups (Max. No) } & Control & 24.45 & 25.8 & 1.35 & 0.76 & 1.77 \\
\hline & & Experimental & 24 & 28.33 & 1.27 & 1.27 & 3.41 \\
\hline \multirow[t]{2}{*}{3} & \multirow{2}{*}{ Chin-ups (Max. No) } & Control & 10.72 & 10.93 & 0.21 & 0.64 & 0.32 \\
\hline & & Experimental & 9.83 & 10.83 & 1 & 0.48 & 2.08 \\
\hline \multirow[t]{2}{*}{4} & \multirow{2}{*}{ Sit-ups (Max. No) } & Control & 51.33 & 56.8 & 5.47 & 3.24 & 1.68 \\
\hline & & Experimental & 52.33 & 58.91 & 6.58 & 1.68 & 3.91 \\
\hline \multirow[t]{2}{*}{5} & \multirow{2}{*}{ Back strength $(\mathrm{kg})$} & Control & 127.1 & 129.4 & 1.33 & 1.92 & 0.69 \\
\hline & & Experimental & 133.7 & 139.5 & 5.83 & 3.28 & 1.77 \\
\hline 6 & $30 \mathrm{M}$ sprint (sec) & Control & 4.66 & 4.55 & 0.11 & 0.12 & 0.91 \\
\hline
\end{tabular}


Citation: Ibrahim S, Hassanain M, Ahmed SA, Ahmed SM, Ahmed SK (2018) Effect of Fitness Training Encompassing Gymnastics-Oriented Program of Six Weeks Duration on Performance of Volleyball Players. J Sports Med Doping Stud 8: 199. doi: 10.4172/2161-0673.1000199

Page 3 of 4

\begin{tabular}{|c|c|c|c|c|c|c|c|}
\hline & & Experimental & 4.62 & 4.53 & 0.09 & 0.07 & 1.28 \\
\hline \multirow[t]{2}{*}{7} & \multirow{2}{*}{ Boomerang (sec) } & Control & 18.22 & 17.3 & 0.92 & 0.51 & 1.8 \\
\hline & & Experimental & 16.83 & 15.42 & 1.41 & 0.35 & 4.02 \\
\hline \multirow[t]{2}{*}{8} & \multirow{2}{*}{$6000 \mathrm{M}$ run (sec) } & Control & 28.29 & 27.99 & 0.3 & 0.22 & 1.5 \\
\hline & & Experimental & 24.81 & 25.13 & 0.32 & 0.4 & 0.8 \\
\hline \multirow[t]{2}{*}{9} & \multirow{2}{*}{ Sit and Reach $(\mathrm{cm})$} & Control & 17.33 & 15.99 & 1.4 & 1.03 & 1.35 \\
\hline & & Experimental & 15.08 & 18.33 & 3.25 & 0.7 & 4.64 \\
\hline \multirow[t]{2}{*}{10} & \multirow{2}{*}{ Bridge up $(\mathrm{cm})$} & Control & 63.6 & 63.47 & 0.13 & 0.92 & 0.14 \\
\hline & & Experimental & 69.96 & 62.58 & 7.38 & 2.08 & 3.55 \\
\hline \multirow[t]{2}{*}{11} & \multirow{2}{*}{$\begin{array}{l}\text { Shoulder rotation (Index } \\
\text { value) }\end{array}$} & Control & 1.85 & 1.88 & 0.03 & 0.06 & 0.5 \\
\hline & & Experimental & 2.13 & 2.06 & 0.03 & 0.03 & 2.33 \\
\hline
\end{tabular}

Table 2: Pre and post T-test values of control group $(n=15)$ and experimental group $(n=12)$ for the physical fitness variables $(x=P<0.05$; $\mathrm{xx}=\mathrm{P}<0.01)$.

Table 2 depicts the means, mean differences, SED and T-Values of the Experimental and Control groups with regard to the physical fitness variables in the pre and the post test scores. It is clearly observed that the training protocol has brought significant improvements in Vertical jump, push-ups, sit-ups, modified boomerang test, bridge test and shoulder rotation index with the $\mathrm{t}$ values being 2.60, 3.41, 3.91, 4.02, 3.55 and 2.33 respectively. However there was no significant difference in the Chin-ups, Back strength, 30 $\mathrm{M}$ sprint and $6000 \mathrm{M}$ run with the t-values being 2.08, 1.77, 1.28 and 0.80 respectively.

\begin{tabular}{|c|c|c|c|c|c|c|c|}
\hline S No. & Variable (Skills) & Group & Pre-Test & Post-Test & Mean & SED \\
\hline & & & Mean \pm SD & Mean \pm SD & Difference & \\
\hline $\mathbf{1}$ & Passing & Control & $7.8 \pm 2.96$ & $9.53 \pm 2.21$ & 1.73 & 0.94 \\
\hline & (Time) & Experimental & $9.75 \pm 2.46$ & $11.83 \pm 3.45$ & 2.08 & 0.57 \\
\hline $\mathbf{2}$ & Spiking & Control & $9.6 \pm 2.14$ & $8.47 \pm 2.62$ & 1.13 & 0.78 \\
\hline & (Max. No) & Experimental & $10.5 \pm 3.08$ & $12.33 \pm 3.96$ & 1.83 & 0.71 & 2.57 \\
\hline $\mathbf{3}$ & Service & Control & $8.13 \pm 2.53$ & $9.76 \pm 2.46$ & 1.63 & 0.74 & 2.20 \\
\hline & (Max. No) & Experimental & $7.92 \pm 2.98$ & $10.42 \pm 3.06$ & 2.5 & 0.69 & 3.62 \\
\hline
\end{tabular}

Table 3: Pre and post test T-values of control group $(n=15)$ and experimental group $(n=12)$ for the skills test scores $(X P<0.05 ; x x P<0.01)$.

Table 3 indicates that there was a significant improvement in the all the post test for the technical skills tests in the experimental group with the T-Values being; $3.65,2.57$, and 3.62 for the passing, spiking and service respectively. There was no significant difference in the values of the control group except in the service where a significant difference was observed.

\section{Discussion}

The main purpose of the study was to find out the effect of gymnastic oriented training program on the physical fitness and the level of technical performance of Volleyball players. It has been found by Nelson et al. [9] that the component of power can be accomplished by tumbling and flexibility exercises. Further, investigations by Hakkinen et al. [10] and Ylinen et al. [11] have shown that stretching is effective as strengthening exercises. The nature of repeated floor jumping exercises apart from vaulting horse and trampoline modalities are explosive and help in arriving at mastering specific movements in various sports. Their administration in the study resulted in significant improvements in the performance of vertical jump in the experimental group as compared to control group.

Parallel bar exercises has been traditionally recognized as the most important in causing changes in the shoulder, chest and arm muscles resulting in the improving the shoulder strength [12]. There was a very high significant improvement in the experimental group in the arms and shoulder strength than the control group. There was an insignificant difference with regard to the pulling ability measured by chin-ups and it can be construed that it takes a longer time to develop pulling strength than the pushing strength. There was a vast improvement in the abdominal strength of the experimental group besides improving the flexibility of the trunk and shoulder. The results of the study with regard to these two particular aspects can be 
Citation: Ibrahim S, Hassanain M, Ahmed SA, Ahmed SM, Ahmed SK (2018) Effect of Fitness Training Encompassing Gymnastics-Oriented Program of Six Weeks Duration on Performance of Volleyball Players. J Sports Med Doping Stud 8: 199. doi: 10.4172/2161-0673.1000199

Page 4 of 4

corroborated with the study of George and Paschalis who had achieved significant results with flexibility exercise and they found that the specific gymnastic oriented training improved the stretchability of muscles and ligaments [6].

The modified boomerang test is a specific test which measures the agility and the coordinative ability [13]. When this test was utilized for the subjects of the experimental group in the study there were outstanding improvements in their agility, coordinative abilities and also the kinesthetic sense. This shows that the tumbling, trampoline and the gymnastics activities have strong relationship with the variables mentioned above.

\section{Conclusion}

It was concluded that the gymnastic oriented exercise program did not have any effect on the anthropometric measurements of the volleyball players. It was also concluded that the experimental group had significant effect on almost all variables under study except the shoulder strength, back strength, speed and endurance.

\section{References}

1. Tsunawake N, Tahara Y, Moji K, Muraki S, Minowa K (2003) Body Composition and Physical Fitness of Female Volleyball and Basketball Players of the Japan Inter-High School Championship Teams. J Physiol Anthropol Appl Human Sci 22: 195-201.

2. Markou KB, Mylonas P, Theodoropoulou A, Kontogiannis A, Leglise M, et al. (2004) The influence of intensive physical exercise on bone acquisition in adolescent elite female and male artistic gymnasts. J Clin Endocrinol Metab 89: 4383-4387.

3. Caine D, Bass S, Daly R (2003) Does elite competition inhibit growth and delay maturation in some gymnasts? Quite possibly. Pediatr Exerc Sci 15: 360-372.
4. Coe DP, Pivarnik JM, Womack CJ, Reeves MJ, Malina RM (2012) Healthrelated fitness and academic achievement in middle school students. J Sports Med Phy Fitness 52: 654-660.

5. Heinen T, Vinken P, Ölsberg P (2010) Manual guidance in gymnastics: A case study, Sci Gym J 2: 043-056.

6. Dallas G, Kirialanis P, Mellos V (2014) The acute effect of whole body vibration training on flexibility and explosive strength of young gymnasts. Biol Sport 31: 233-237.

7. Silva M, Lacerda D, João PV (2014) Game-related volleyball skills that influence victory. J Hum Kinet 41: 173-179.

8. Cormie P, Deane RS, Triplett NT, McBride JM (2006) Acute effects of whole-body vibration on muscle activity, strength, and power. J Strength Cond Res 20: 257-261.

9. Nelson AG, Kokkonen J, Arnall DA (2005) Acute muscle stretching inhibits muscle strength endurance performance. J Strength Cond Res 19: 338-343.

10. Hakkinen A, Kautiainen H, Hannonen P, Ylinen J (2008) Strength training and stretching versus stretching only in the treatment of patients with chronic neck pain: A randomized one-year follow-up study. Clin Rehabil 22: 592-600.

11. Ylinen J, Kautiainen H, Wirén K, Häkkinen A (2007) Stretching exercises vs manual therapy in treatment of chronic neck pain: A randomized, controlled cross-over trial. J Rehabil Med 39: 126-132.

12. Velickovic S, Paunovic M, Vukasinovic V (2016) Systematization of previous researches of exercises on parallel bars. Facta Universitatis Series: Physical Education and Sport 14: 51-62.

13. Byrne S, Linz D, Potter WJ (2009) A test of competing cognitive explanations for the boomerang effect in response to the deliberate disruption of media-induced aggression. Media Psychol 12: 227-248. 